\title{
Fluid-structure coupling by the entrained fluid in submerged concentric double-shell vibration
}

\author{
Shigeru Yoshikawa \\ 5th Research Center, Technical $R \&$ D Institute, Defense Agency, \\ 3-13-1 Nagase, Yokosuka, 239 Japan
}

(Received 28 July 1992)

\begin{abstract}
Vibrational characteristics of a point-driven "double shell" (two concentric submerged cylindrical shells coupled by the entrained fluid) are investigated. Strong- and weakcoupling theories are proposed to model the interaction of how the inner and outer shells are coupled by the entrained fluid. Each theory is based on Flügge's infinite-shell equations, the Helmholtz wave equation, and boundary conditions at the fluid-structure interfaces which depend on the coupling situation. Strong coupling forms a standing wavefield in the entrained fluid; weak coupling, a free wavefield. Experiments are carried out where generalized near-field acoustical holography (GENAH) is employed to provide the experimental vibration characteristics in wavenumber-frequency space of the finite double-shell. It is confirmed theoretically and experimentally that the outer shell of the double shell exhibits two separate dispersion curves: A higher-frequency curve bears in-phase vibrations with respect to the inner shell and suggests a forced response of the inner shell; the other lower-frequency curve, out-of-phase vibrations, and an induced response of the outer shell. Fluid-structure coupling condition definitely affects the lower-frequency curve, but barely the higher-frequency one. Comparison between theoretical and experimental results demonstrates the validity of the strong-coupling model.
\end{abstract}

Keywords: Underwater structural vibration, Fluid-structure interaction, Double shell, Entrained fluid, K-space analysis, Propagator, Dispersion curve, Generalized near-field acoustical holography

PACS number: 43. 40. At, 43. 40. Ey, 43. 20. Tb, 43. 60. Sx

\section{INTRODUCTION}

Sound radiation of very low frequencies from ships and submersibles is presumably caused by the overall vibration of their whole or central body. Manifest evidences of such vibration or radiation, however, have not yet obtained experimentally or theoretically. This is because real ships take shapes and structures of large scale and high complexity. It should be thus recognized that present available theories ${ }^{1,2)}$ applicable to the research of ship vibration and radiation are far from reality, particularly in low frequency region. Also, it will be profitless so far to resort to the computer simulation (based on the finite element and/or boundary element method) without promoting proper theoretical or experimental investigations because there still remains many problems in modeling itself. ${ }^{3)}$ For example, we do not know exactly how to model the ribbed structures for ship-hull reinforcement, the discontinuity on hull plates, and the fluid-structure interaction through the entrained fluid in double-hull configuration. The research of these major contributing factors will thus take priority over that of the whole in order to construct ship structural acoustics.

As an example of such factor research, this paper is then focusing on the vibroacoustical role of the fluid entrained between two concentric submerged 
cylindrical shells (the shells having a configuration like this will be referred to as a double shell below). Double-hull structure of submersibles is here simplified as a cylindrical double shell without ribbs. Such a simplification will make it possible to create fundamental theory on the interaction among the inner shell, the outer shell, the entrained fluid, and the surrounding fluid.

From the viewpoint of vibroacoustical differences between single and double shells immersed in fluid, the coupled vibration of a double shell was already studied theoretically and experimentally. ${ }^{4-7)}$ Then, only brief outline of Flügge's shell equations and their K-space (i.e., spatial wavenumber/frequencyspace) representation will be given in Section 3 after the description of our point-driven double-shell model in Section 2. Since acoustic pressure may propagate from the inner shell to the outer shell through the annular fluid space between them, the Helmholtz wave equation is applicable to this field (Section 4). The acoustic pressure in this internal field exerts fluid-loading force to the enclosing shells, whose strength depends on the coupling between the fluid field and shell structure. Theories on strong and weak couplings will be described in Section 5 , from which $6 \times 6$ matrix equation with fluid-structure coupling elements will be derived in Section 6 and numerically calculated in Section 7. Experimental results obtained from the GENAH (GEneralized Near-field Acoustical Holography) measurement $t^{8,9)}$ on a double-shell model immersed in water pool will be explained in Section 8. Theoretical and experimental results, which are expressed as dispersion curve given by $\mathrm{K}$-space analysis, will be compared with each other to examine which coupling is predominant (Section 9). In conclusion, vibrational behaviors due to the fluid-structure coupling will be summarized.

\section{MODEL DESCRIPTION}

The coupled vibration of a theoretical model consisting of two concentric circular cylindrical shells (a double shell, see Fig. 1) is considered in this paper. The inner shell contains air, the outer shell is surrounded by fluid, and the annular space between them is occupied by fluid. This annular fluid is referred to as the entrained fluid. The inner and outer shells are vibroacoustically coupled by this entrained fluid. The objective of this paper is to consider what kind of fluid-structure interaction is
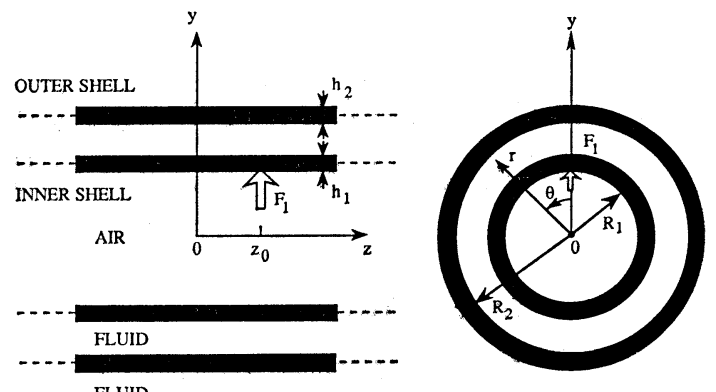

FLUID

Fig. 1 Theoretical model of the double shell. The shell is infinite and driven by a point force $F_{1}$ at $r=R_{1}, \theta=0$, and $z=z_{0}$. The inner shell contains air, the outer shell is surrounded by fluid, and the annular space between them is occupied by fluid.

\section{occurring among them.}

Theoretical double-shell model of Fig. 1 is infinite and driven radially with a point force $F_{1}$ located at $r=R_{1}, \theta=0$, and $z=z_{0}$ in the cylindrical coordinate system $(r, \theta, z)$. The force is harmonic and has the time dependence of $\exp (-i \omega t)$, where $\omega$ is the angular frequency, $t$ the time, and $i$ the square root of -1 . Shell thickness and mean radius are denoted by $h$ and $R$, respectively. Subscript $i$ is added to those symbols to discriminate the inner shell $(i=1)$ from the outer one $(i=2)$. Each shell is made of the same material and we denote its density, Young's modulus, and Poisson's ratio by $\rho, E$, and $\nu$, respectively.

Experimental model corresponding to the theoretical one shown in Fig. 1 is also constructed (see Fig. 2). Its dimensions and material constants are shown in Table 1. The inner shell is closed by disk endcaps. These endcaps are supported by three rods which go through the holes arranged on the disk endcap. Three small holes are perforated in each ring endcap (not shown in Fig. 2) to conduct water into the annular space between the inner and outer shells. Rubber packings are used to obtain waterproofness of the inner shell and to give the outer shell the boundary condition similar to that of the inner shell. An O-ring is, moreover, used for a lid that screws on a supporting rod to assure waterproofness. The boundary condition at the shell end may be considered as simply-supported. A small and lightweight piezoelectric shaker is attached on the inner shell. The position of this shaker is 


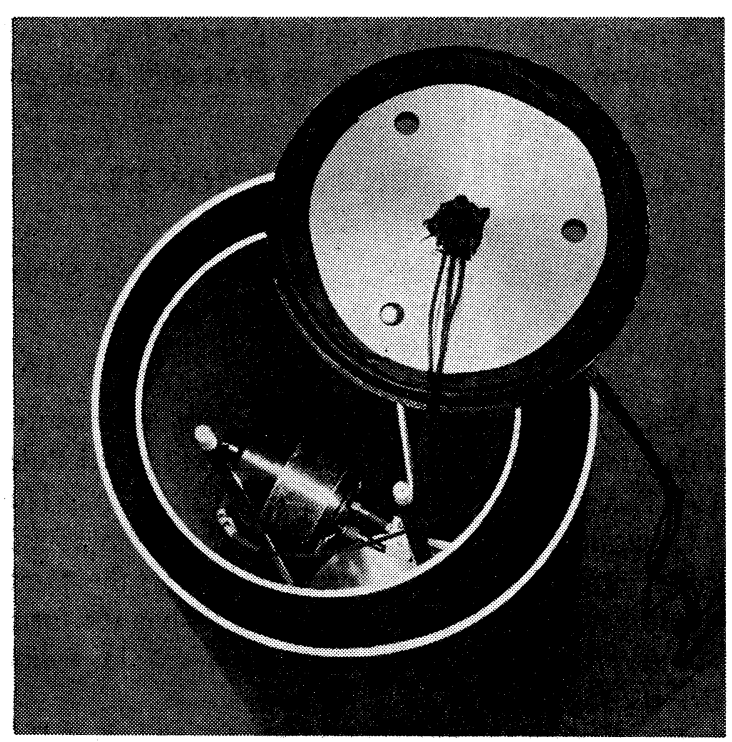

Fig. 2 Experimental model of the double shell. The shell is finite and simply-supported by the endcaps with rubber packings. A small shaker is provided as a harmonic point-force driver.

Table 1 Numerical values of the experimental double-shell model.

\begin{tabular}{lll}
\hline Inner Shell & & stainless steel \\
Density & $\rho\left(\mathrm{kg} / \mathrm{m}^{3}\right)$ & $7.93 \times 10^{3}$ \\
Young's modulus & $E(\mathrm{~N} / \mathrm{m})$ & $1.95 \times 10^{11}$ \\
Poisson's ratio & $\nu$ & 0.28 \\
Loss factor & $\eta$ & 0.001 \\
\hline Mean radius & $R_{1}(\mathrm{~m})$ & 0.081 \\
Thickness & $h_{1}(\mathrm{~m})$ & 0.003 \\
Length & $l(\mathrm{~m})$ & 0.800 \\
\hline Outer Shell & & stainless steel \\
Mean radius & $R_{2}(\mathrm{~m})$ & 0.107 \\
Thickness & $h_{2}(\mathrm{~m})$ & 0.003 \\
Length & $l(\mathrm{~m})$ & 0.800 \\
\hline Fluid & & water \\
Density & $\rho_{0}\left(\mathrm{~kg} / \mathrm{m}^{3}\right)$ & $1.0 \times 10^{3}$ \\
Sound speed & $c_{0}(\mathrm{~m} / \mathrm{s})$ & $1.4883 \times 10^{3}$ \\
\hline Driver & & harmonic \\
Position & & point-force \\
& $z_{0}(\mathrm{~m})$ & 0.1 (from the \\
\hline
\end{tabular}

$0.10 \mathrm{~m}$ up from the shell center. The shaker can include a force gauge to measure the force exerted on the shell.
Finite-shell vibration shows the spatial periodicity distributed along the shell axis and has discrete axial wavenumber spectrum; infinite-shell vibration shows the spatial nonperiodicity and has continuous axial wavenumber spectrum. The simply-supported boundary condition for the finite shell is expressed as

$$
k_{z} l=m \pi,
$$

where $k_{z}$ is the axial wavenumber, $m$ the axial mode number (the number of half-wavelength along the shell axis, $m=1,2,3, \ldots)$, and $l$ the shell length. These $k_{z}$ and $m$ are discrete for finite shells, while they should be treated as continuous for infinite shells. However, if we consider an infinite shell with an axially periodic distribution of vibration restricted to a finite part of shell which satisfies Eq. (1), it may correspond to the finite-shell vibration. ${ }^{10}$ ) Therefore, the comparison between theory on an infinite shell and experiment on a finite shell is safely allowed.

\section{INFINITE-SHELL EQUATIONS AND THEIR K-SPACE REPRESENTATION}

Brief outline of infinite-shell equations and their $\mathrm{K}$-space representation is described in this section. See Ref. 4) for more details.

The equation of motion of the shell $(i=1,2)$ shown in Fig. 1 can be written as ${ }^{11)}$

$$
\begin{aligned}
\left(\begin{array}{ccc}
l_{i 1} & l_{i 2} & l_{i 3} \\
l_{i 2} & l_{i 4} & l_{i 5} \\
l_{i 3} & l_{i 5} & l_{i 6}
\end{array}\right)\left(\begin{array}{c}
u_{i} \\
v_{i} \\
w_{i}
\end{array}\right) \\
\quad=\frac{R_{i}^{2}\left(1-\nu^{2}\right)}{E h_{i}}\left(\begin{array}{c}
0 \\
0 \\
-q_{i}+F_{i} \delta\left(z-z_{0}\right) \delta(\theta) / R_{i}
\end{array}\right),
\end{aligned}
$$

where the axial, circumferential, and radial displacements of the shell are given by $u_{i}, v_{i}, w_{i}$, respectively. The quantity $q_{i}$ is the acoustic pressure loading on the cylinder. Since the driving force is applied to the inner shell only, $F_{2}$ equals zero. According to our convention, $F_{1}$ is defined as positive outward, while $q_{i}$ is defined as positive inward. The $\delta$ is the Dirac delta function. Subscript $i$ of the matrix elements $l_{i j}(j=1,2,3,4,5,6)$ takes 1 for the inner shell and 2 for the outer one. These $l_{i j}$ are differential operators with values specified by the particular shell theory used. For Flügge's theory, ${ }^{11)}$ for example, 


$$
\begin{aligned}
l_{i 6}= & +\left(\frac{h_{i}{ }^{2}}{12 R_{i}{ }^{2}}\right)\left[1+R_{i}{ }^{4}\left(\frac{\partial^{4}}{\partial z^{4}}\right)+2 R_{i}{ }^{2}\left(\frac{\partial^{4}}{\partial z^{2} \partial \theta^{2}}\right)\right. \\
& \left.+\left(\frac{\partial^{4}}{\partial \theta^{4}}\right)+2\left(\frac{\partial^{2}}{\partial \theta^{2}}\right)\right]+\left(\frac{R_{i}{ }^{2}}{c_{p}{ }^{2}}\right)\left(\frac{\partial^{2}}{\partial t^{2}}\right),
\end{aligned}
$$

where the quantity $c_{p}\left[=\sqrt{E / \rho\left(1-\nu^{2}\right)}\right]$ for $i=1$ and 2 is the compressional wave speed in both inner and outer shells.

Equation (2) is transformed to algebraic equations by application of the two-dimensional, spatial Fourier transform:

$$
\begin{aligned}
& \left(\begin{array}{lll}
L_{i 1} & L_{i 2} & L_{i 3} \\
L_{i 2} & L_{i 4} & L_{i 5} \\
L_{i 3} & L_{i 5} & L_{i 6}
\end{array}\right)\left(\begin{array}{c}
U_{i n} \\
V_{i n} \\
W_{i n}
\end{array}\right) \\
& =\frac{R_{i}^{2}\left(1-\nu^{2}\right)}{E h_{i}}\left(\begin{array}{c}
0 \\
0 \\
-Q_{i n}+\frac{F_{i} \exp \left(-i k_{z} z_{0}\right)}{2 \pi R_{i}}
\end{array}\right),
\end{aligned}
$$

where $L_{i j}, Q_{i n}, U_{i n}, V_{i n}$, and $W_{i n}$ represent the transforms of $l_{i j}, q_{i}, u_{i}, v_{i}$, and $w_{i}$, respectively. For example, $W_{i n}$ is defined as

$$
W_{i n}\left(k_{z}, \omega\right)=\frac{1}{2 \pi} \int_{0}^{2 \pi} d \theta e^{-i n \theta} \int_{-\infty}^{\infty} d z e^{-i k_{z} z} w_{i}(\theta, z, \omega),
$$

where $n$ represents the circumferential wavenumber. Corresponding to the above $l_{i 6}$, we get the following:

$$
\begin{aligned}
L_{i 6}= & 1-\Omega_{i}{ }^{2}+\left[\left(\frac{1}{12}\right)\left(\frac{h_{i}}{R_{i}}\right)^{2}\right] \\
& \cdot\left\{1-2 n^{2}+\left[\left(k_{z} R_{i}\right)^{2}+n^{2}\right]^{2}\right\},
\end{aligned}
$$

where $\Omega_{i}$ is the nondimensional frequency defined as

$$
\Omega_{i}=\frac{R_{i}}{c_{p}} \omega .
$$

The expression of the inverse transform of Eq. (4) gives a decomposition of the radial surface displacement in terms of helical waves, i.e., $W_{i n}\left(k_{z}, \omega\right)$ $\exp \left[i\left(k_{z} z+n \theta-\omega t\right)\right]$. Since these components make up the helical-wave or $\mathrm{K}$-space spectrum, we will refer to Eq. (3) as the K-space representation of the shell equations. Also, we will refer to the complex amplitude $W_{i n}\left(k_{z}, \omega\right)$ as wavenumber-frequency (or $k-\omega)$ representation of shell displacement $w_{i}(z, \theta, \omega)$ when we consider the $\mathrm{K}$-space spectrum as a function of $k_{z}$ and $\omega$ by fixing $n$. Theory and experiment described below are based on this $k-\omega$ representation. Major advantages of this representation are that i) the algebraic form of Eq. (3) is handl- ed more easily than the operator form of Eq. (2) and ii) advanced signal processings are easily available in $k-\omega$ space.

\section{ACOUSTIC PROPAGATION IN FLUID FIELD}

Since the acoustic wave propagates in fluid fields, we may apply the Helmholtz equation to them:

$$
\nabla^{2} p_{j}+k_{0}^{2} p_{j}=0
$$

where $p_{j}(r, \theta, z, \omega)$ expresses the acoustic pressure. The subscript $j$ denotes the annular entrained fluid $(j=1)$ and the surrounding free fluid $(j=2)$. The wavenumber $k_{0}$ is defined as $\omega / c_{0}$ using the sound speed $c_{0}$ in the fluid (we are considering the same fluid in two fields). The Laplacian $\nabla^{2}$ has the form $(1 / r)(\partial / \partial r)(r \partial / \partial r)+\left(1 / r^{2}\right)\left(\partial^{2} / \partial \theta^{2}\right)+\left(\partial^{2} / \partial z^{2}\right)$ in cylindrical coordinates.

If we apply the spatial Fourier transform of Eq. (4) to Eq. (6), we have the following Bessel's equation:

$$
\left(\xi \frac{d}{d \xi}\right)^{2} P_{j n}+\left(\xi \frac{d}{d \xi}\right) P_{j n}+\left(\xi^{2}-n^{2}\right) P_{j n}=0,
$$

where $P_{j n}\left(r, k_{z}, \omega\right)$ corresponds to $k-\omega$ representation of $p_{j}$, and $\xi=r \sqrt{k_{0}^{2}-k_{z}^{2}}$. This Eq. (7) gives. the general solution

$$
P_{j n}(\xi)=a_{j} A_{j n}(\xi)+b_{j} B_{j n}(\xi),
$$

where

$$
\begin{gathered}
A_{j n}(\xi)= \begin{cases}J_{n}\left(k_{r} r\right) & c>c_{0} \\
I_{n}\left(k_{r}^{\prime} r\right) & c \leq c_{0},\end{cases} \\
B_{j n}(\xi)= \begin{cases}Y_{n}\left(k_{r} r\right) & c>c_{0} \\
K_{r}\left(k_{r}{ }^{\prime} r\right) & c \leq c_{0},\end{cases} \\
k_{r}=\sqrt{k_{0}^{2}-k_{z}^{2}}=k_{z} \sqrt{\left(c / c_{0}\right)^{2}-1}, \\
k_{r}{ }^{\prime}=\sqrt{k_{z}^{2}-k_{0}^{2}}=k_{z} \sqrt{1-\left(c / c_{0}\right)^{2}}, \\
k_{z}=\frac{\omega}{c}, \\
k_{0}=\frac{\omega}{c_{0}} .
\end{gathered}
$$

The quantity $c$ in Eqs. (9) to (13) means the phase velocity of the helical wave propagating in the axial direction. Functions $J_{n}$ and $Y_{n}$, which are the Bessel's functions of the first and second kind respectively, are used in the supersonic region $c>c_{0}$, while $I_{n}$ and $K_{n}$, which are the modified Bessel's functions, are used in the subsonic region $c \leq c_{0}$. The quantities $k_{r}$ and $k_{r}^{\prime}$ are the radial wavenumber in 


\section{S. YOSHIKAWA: FLUID-STRUCTURE COUPLED VIBRATION OF DOUBLE SHELL}

these regions, respectively. The quantities $k_{0}$ and $k_{z}$ are the acoustic wavenumber of fluid and the axial wavenumber of shell, respectively.

Coefficients $a_{j}$ and $b_{j}$ of Eq. (8) are determined by the boundary conditions at the fluid-structure interfaces and the radiation condition for the free field. Since there are four unknown coefficients $a_{1}, a_{2}, b_{1}$, and $b_{2}$, we have to prepare four equations describing boundary and radiation conditions to determine them. It will be carried out in the next section.

\section{FLUID-STRUCTURE COUPLING}

There seems to be two kinds of possibility on the vibroacoustical function of the entrained fluid.

Strong Coupling: The forced vibration of the inner shell propagates to the outer shell through the entrained fluid. A part of the incidental energy of this vibratory wave drives the outer shell, and the rest of it is reflected back to the inner shell. The induced vibration of the outer shell radiates sound into the free field; the reflected wave affects the original forced vibration of the inner shell in a degree. In other words, we may say that a standing wavefield is formed in the entrained fluid. Strongly coupled vibration of the double shell will thus take place.

Weak Coupling: However, if the reflection at the outer shell is weak because of small difference in mechanical impedance between the entrained fluid and outer shell, or if the driving force applied to the inner shell is so sturdy that the reflected wave gives only negligible contribution to the inner shell, the coupling will become weak. The outer shell is still driven by the acoustic wave from the inner shell and radiates sound toward the free field, while the inner shell may behave like an independent vibrator because it will be unaffected by the presence of the entrained fluid and outer shell. In other words, we may say that a free wavefield is formed in the entrained fluid. Weakly coupled vibration of the double shell will thus take place.

Much simpler examples illustrating the abovementioned concepts of strong and weak couplings are found in Ref. 12), where idealized models of i) sound transmission through a double-leaf partition and ii) cavity wavefield between a vibrating surface and its cladding enclosure to reduce sound radiation are considered. For more complete treatment, the theories described below should be applied to them.
5.1 K-space Fluid Loadings due to Strong Coupling

The boundary conditions are given by the continuity of radial velocity at the fluid-shell interfaces:

$$
\begin{aligned}
& \dot{w}_{1}=\left(\frac{1}{i \omega \rho_{0}}\right)\left(\frac{\partial p_{1}}{\partial r}\right)_{r=R_{1}}, \\
& \dot{w}_{2}=\left(\frac{1}{i \omega \rho_{0}}\right)\left(\frac{\partial p_{1}}{\partial r}\right)_{r=R_{2}}=\left(\frac{1}{i \omega \rho_{0}}\right)\left(\frac{\partial p_{2}}{\partial r}\right)_{r=R_{2}}
\end{aligned}
$$

where the dot denotes the time derivative. In $\mathrm{K}$ space, by applying Eqs. (4) and (8) to Eq. (15), we obtain respectively

$$
\begin{aligned}
& a_{1} A_{1 n}{ }^{\prime}\left(R_{1}\right)+b_{1} B_{1 n}{ }^{\prime}\left(R_{1}\right)=\omega^{2} \rho_{0} W_{1 n}, \\
& a_{1} A_{1 n}{ }^{\prime}\left(R_{2}\right)+b_{1} B_{1 n}{ }^{\prime}\left(R_{2}\right)=\omega^{2} \rho_{0} W_{2 n}, \\
& a_{2} A_{2 n}{ }^{\prime}\left(R_{2}\right)+b_{2} B_{2 n}{ }^{\prime}\left(R_{2}\right)=\omega^{2} \rho_{0} W_{2 n},
\end{aligned}
$$

where the prime denotes differentiation with respect to $r$. The radiation condition requires that the transformed acoustic pressure $P_{2 n}(r)$ in the free field $(j=2)$ must have the form of outgoing wave, i.e., the Hankel function $H_{n}^{(1)}(r)\left(c>c_{0}\right)$ or the modified Bessel function $K_{n}(r)\left(c \leq c_{0}\right)$. This means that

$$
b_{2}=i a_{2}\left(c>c_{0}\right), \quad a_{2}=0\left(c \leq c_{0}\right) .
$$

The above four equations of Eqs. (16) and (17) determine $a_{1}, a_{2}, b_{1}$, and $b_{2}$ as functions of $W_{1 n}$ and $W_{2 n}$. Finally, we can obtain the following solution of the transformed acoustic pressure:

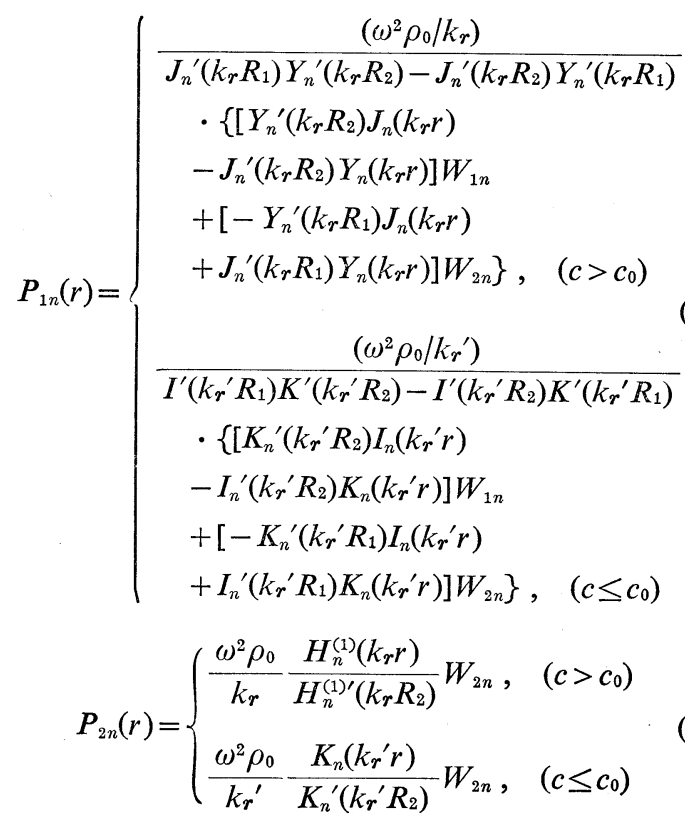


where the prime defines differentiation with respect to the argument $\left(k_{r} r\right.$ or $\left.k_{r}{ }^{\prime} r\right)$ of the Bessel functions.

Fluid (or acoustic) loadings involved in Eq. (2) are given by

$$
q_{1}=\left(p_{1}\right)_{r=R_{1}}, \quad q_{2}=\left(p_{2}-p_{1}\right)_{r=R_{2}} .
$$

By inserting the solution given as Eqs. (18) and (19) into the K-space representation of Eq. (20), we can obtain the corresponding $\mathrm{K}$-space fluid loadings as follows:

$$
\begin{aligned}
& Q_{1 n}\left(k_{z}, \omega\right)=\left[G_{12}\left(R_{1}\right) W_{1 n}\left(k_{z}, \omega\right)+G_{21}\left(R_{1}\right) W_{2 n}\left(k_{z}, \omega\right)\right], \\
& Q_{2 n}\left(k_{z}, \omega\right)=-\left[G_{12}\left(R_{2}\right) W_{1 n}\left(k_{z}, \omega\right)\right. \\
& + \\
& \left.+G_{21}\left(R_{2}\right) W_{2 n}\left(k_{z}, \omega\right)\right] \\
& +G_{22}\left(R_{2}\right) W_{2 n}\left(k_{z}, \omega\right),
\end{aligned}
$$

where

$$
\begin{aligned}
& G_{12}(r)=\left\{\begin{array}{c}
\left(\frac{\omega^{2} \rho_{0}}{k_{r}}\right) \\
\frac{J_{n}\left(k_{r} r\right) Y_{n}^{\prime}\left(k_{r} R_{2}\right)-Y_{n}\left(k_{r} r\right) J_{n}^{\prime}\left(k_{r} R_{2}\right)}{J_{n}^{\prime}\left(k_{r} R_{1}\right) Y_{n}^{\prime}\left(k_{r} R_{2}\right)-Y_{n}^{\prime}\left(k_{r} R_{1}\right) J_{n}{ }^{\prime}\left(k_{r} R_{2}\right)} \\
\left(c>c_{0}\right)
\end{array}\right. \\
& \left(\frac{\omega^{2} \rho_{0}}{k_{r}{ }^{\prime}}\right) \\
& \begin{array}{c}
\cdot \frac{I_{n}\left(k_{r}^{\prime} r\right) K_{n}{ }^{\prime}\left(k_{r}{ }^{\prime} R_{2}\right)-K_{n}\left(k_{r}{ }^{\prime} r\right) I_{n}{ }^{\prime}\left(k_{r}{ }^{\prime} R_{2}\right)}{I_{n}\left(k_{r}^{\prime} R_{1}\right) K_{n}{ }^{\prime}\left(k_{r}{ }^{\prime} R_{2}\right)-K_{n}{ }^{\prime}\left(k_{r}^{\prime} R_{1}\right) I_{n}{ }^{\prime}\left(k_{r}{ }^{\prime} R_{2}\right)}, \\
\left(c \leq c_{0}\right)
\end{array}
\end{aligned}
$$

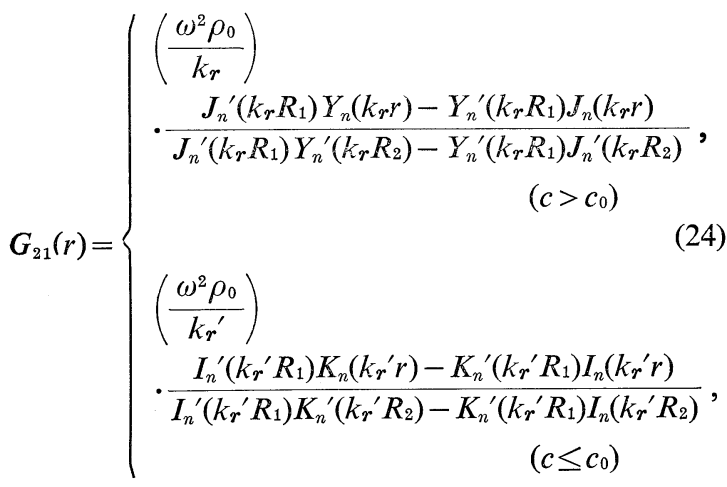

$$
\begin{aligned}
& G_{22}(r)= \begin{cases}\left(\frac{\omega^{2} \rho_{0}}{k_{r}}\right) \frac{H_{n}^{(1)}\left(k_{r} r\right)}{H_{n}^{(1)}\left(k_{r} R_{2}\right)} \cdot & \left(c>c_{0}\right) \\
\left(\frac{\omega^{2} \rho_{0}}{k_{r}^{\prime}}\right) \frac{K_{n}\left(k_{r}^{\prime} r\right)}{K_{n}^{\prime}\left(k_{r}^{\prime} R_{2}\right)} \cdot, \quad\left(c \leq c_{0}\right)\end{cases}
\end{aligned}
$$

The quantities $G_{12}(r), G_{21}(r)$, and $G_{22}(r)$ defined by (a) [STRONG COUPLING]

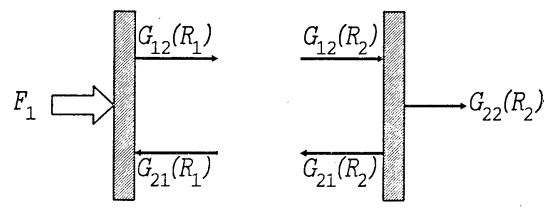

INNER SHELL OUTER SHELL

(b)

[WEAK COUPLING]

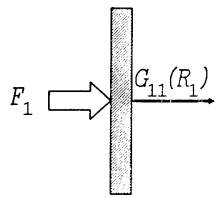

INNER SHELL

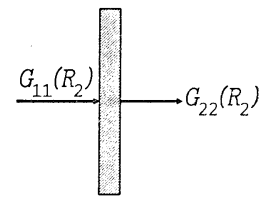

OUTER SHELL
Fig. 3 Fluid-structure coupling in pointdriven, double-shell vibration on the basis of $\mathrm{K}$-space representation. (a) strong coupling, (b) weak coupling.

Eqs. (23), (24), and (25) respectively, may be called propagators: $G_{12}(r)$ describes the propagation from the inner shell to the outer shell; $G_{21}(r)$ the propagation from the outer shell to the inner shell; and $G_{22}(r)$ the radiation from the outer shell to the surrounding free field. Figure 3(a) illustrates the strong fluidstructure coupling defined by these propagators.

5.2 K-space Fluid Loadings due to Weak Coupling Weakly coupled vibration will happen if a free wavefield instead of a standing wavefield may be formed in the entrained fluid. Schematically, this is done by ignoring the propagator $G_{21}(r)$ in Fig. 3(a). Mathematically, this means that the radiation condition is satisfied for the entrained fluid as well as for the surrounding fluid:

$$
b_{1}=i a_{1}\left(c>c_{0}\right), \quad a_{1}=0\left(c \leq c_{0}\right) .
$$

Also, the second equation of Eq. (16) should be eliminated because the motion of outer shell $w_{2}$ does not contribute to the acoustic pressure $p_{1}$ in the entrained fluid if the free wavefield is assumed there. Hence, we get two boundary conditions [the first and third equations of Eq. (16)] and two radiation conditions [Eqs. (17) and (26)] to determine unknown coefficients $a_{1}, a_{2}, b_{1}$, and $b_{2}$. From these conditions we obtain the following expression of the transformed pressure in K space instead of Eq. (18): 


\section{S. YOSHIKAWA: FLUID-STRUCTURE COUPLED VIBRATION OF DOUBLE SHELL}

$$
P_{1 n}(r)= \begin{cases}\frac{\omega^{2} \rho_{0}}{k_{r}} \frac{H_{n}^{(1)}\left(k_{r} r\right)}{H_{n}^{(1)}\left(k_{r} R_{1}\right)} W_{1 n}, & \left(c>c_{0}\right) \\ \frac{\omega^{2} \rho_{0}}{k_{r}} \frac{K_{n}\left(k_{r}{ }^{\prime} r\right)}{K_{n}{ }^{\prime}\left(k_{r}{ }^{\prime} R_{1}\right)} W_{1 n}, & \left(c \leq c_{0}\right)\end{cases}
$$

The transformed pressure $P_{2 n}(r)$ in the surrounding free wavefield is given by the previous Eq. (19).

The corresponding K-space fluid loadings are obtained by inserting Eqs. (19) and (27) into Eq. (20):

$$
\begin{aligned}
Q_{1 n}\left(k_{z}, \omega\right)= & G_{11}\left(R_{1}\right) W_{1 n}\left(k_{z}, \omega\right) \\
Q_{2 n}\left(k_{z}, \omega\right)= & -G_{11}\left(R_{2}\right) W_{1 n}\left(k_{z}, \omega\right) \\
& +G_{22}\left(R_{2}\right) W_{2 n}\left(k_{z}, \omega\right)
\end{aligned}
$$

where the propagator

$$
G_{11}(r)= \begin{cases}\left(\frac{\omega^{2} \rho_{0}}{k_{r}}\right) \frac{H_{n}^{(1)}\left(k_{r} r\right)}{H_{n}^{(1)}\left(k_{r} R_{1}\right)} \cdot & \left(c>c_{0}\right) \\ \left(\frac{\omega^{2} \rho_{0}}{k_{r}^{\prime}}\right) \frac{K_{n}\left(k_{r}^{\prime} r\right)}{K_{n}^{\prime}\left(k_{r}^{\prime} R_{1}\right)} \cdot, \quad\left(c \leq c_{0}\right)\end{cases}
$$

The propagator $G_{22}(r)$ was already given by Eq. (25). Figure $3(\mathrm{~b})$ illustrates the weak coupling defined by these two propagators.

\section{6. $6 \times 6$ MATRIX EQUATION WHTH FLUDD-STRUCTURE COUPLING ELEMENTS}

6.1 Strongly-Coupled Vibration of the Double Shell

Substituting $Q_{i n}(i=1,2)$ given by Eqs. (21) and (22) for those in Eq. (3), we have the following $6 \times 6$ matrix equation of double-shell vibration:

$$
\begin{aligned}
& \left(\begin{array}{cccccc}
L_{11} & L_{12} & L_{13} & 0 & 0 & 0 \\
L_{12} & L_{14} & L_{15} & 0 & 0 & 0 \\
L_{13} & L_{15} & L_{16}+L_{16 \mathrm{~F}} & 0 & 0 & L_{17 \mathrm{~F}} \\
0 & 0 & 0 & L_{21} & L_{22} & L_{23} \\
0 & 0 & 0 & L_{22} & L_{24} & L_{25} \\
0 & 0 & L_{27 \mathrm{~F}} & L_{23} & L_{25} & L_{26}+L_{26 \mathrm{~F}}
\end{array}\right)\left(\begin{array}{c}
U_{1 n} \\
V_{1 n} \\
W_{1 n} \\
U_{2 n} \\
V_{2 n} \\
W_{2 n}
\end{array}\right) \\
& =\left(\begin{array}{c}
0 \\
0 \\
F_{1}^{\prime} \\
0 \\
0 \\
0
\end{array}\right),
\end{aligned}
$$

where

$$
\begin{gathered}
L_{16 \mathrm{~F}}=D_{1} G_{12}\left(R_{1}\right), \\
L_{17 \mathrm{~F}}=D_{1} G_{21}\left(R_{1}\right), \\
L_{26 \mathrm{~F}}=D_{2}\left[G_{22}\left(R_{2}\right)-G_{21}\left(R_{2}\right)\right], \\
L_{27 \mathrm{~F}}=-D_{2} G_{12}\left(R_{2}\right), \\
F_{1}^{\prime}=D_{1} F_{1} \exp \left(-i k_{z} z_{0}\right) /\left(2 \pi R_{1}\right), \\
D_{i}=\left(1-\nu^{2}\right) R_{i}{ }^{2} /\left(E h_{i}\right) \quad(i=1,2), \\
\alpha_{i}=\rho_{0} R_{i} / \rho h_{i} \quad(i=1,2) .
\end{gathered}
$$

Equations (5), (23), (24), and (25) are employed to get the explicit, nondimensionalized forms of Eqs. (32) to (35). The matrix elements $L_{16 \mathrm{~F}}, L_{17 \mathrm{~F}}$, $L_{26 \mathrm{~F}}$, and $L_{27 \mathrm{~F}}$ indicate strong fluid-structure coupling in our immersed double shell. Particularly, $L_{17 \mathrm{~F}}$ and $L_{27 \mathrm{~F}}$ couple the inner and outer shells to each other via the entrained fluid.

The radial displacements $W_{1 n}$ and $W_{2 n}$ in $\mathrm{K}$ space are expressed as follows by solving Eq. (31):

$$
\begin{aligned}
& W_{1 n}\left(k_{z}, \omega\right)=\left|\boldsymbol{L}^{\prime}\right| /|\boldsymbol{L}|, \\
& W_{2 n}\left(k_{z}, \omega\right)=\left|\boldsymbol{L}^{\prime \prime}\right| /|\boldsymbol{L}|,
\end{aligned}
$$

where the matrix $\mathbb{L}$ denotes $6 \times 6$ matrix in the left side of Eq. (31) and its determinant is given by

$$
|\mathbb{L}|=\left|L_{1}\right|\left|L_{2}\right|-L_{17 \mathrm{~F}} L_{27 \mathrm{~F}}\left|\begin{array}{ll}
L_{11} & L_{12} \\
L_{12} & L_{14}
\end{array}\right| \cdot\left|\begin{array}{ll}
L_{21} & L_{22} \\
L_{22} & L_{24}
\end{array}\right| .
$$

In Eq. (41) $L_{1}$ is $3 \times 3$ matrix of the immersed inner shell alone and defined by nine elements of row 1 to 3 and line 1 to 3 in $\boldsymbol{L}$. Similarly, $\boldsymbol{L}_{2}$ is that of the immersed outer shell alone and defined by nine elements of row 4 to 6 and line 4 to 6 in $L$. Determinants $\left|L^{\prime}\right|$ and $\left|\boldsymbol{L}^{\prime \prime}\right|$ in Eqs. (39) and (40) are given by

$$
\begin{gathered}
\left|\boldsymbol{L}^{\prime}\right|=F_{1}^{\prime}\left|\boldsymbol{L}_{2}\right| \cdot\left|\begin{array}{ll}
L_{11} & L_{12} \\
L_{12} & L_{14}
\end{array}\right|, \\
\left|\boldsymbol{L}^{\prime \prime}\right|=-F_{1}^{\prime} L_{27 \mathrm{~F}}\left|\begin{array}{ll}
L_{11} & L_{12} \\
L_{12} & L_{14}
\end{array}\right| \cdot\left|\begin{array}{ll}
L_{21} & L_{22} \\
L_{22} & L_{24}
\end{array}\right|,
\end{gathered}
$$

respectively.

6.2 Weakly-Coupled Vibration of the Double Shell

We can obtain similar expressions for the weak coupling by using Eqs. (28) and (29) instead of Eqs. (21) and (22). We then have to employ the following forms of coupling elements in Eq. (31):

$$
\begin{aligned}
& L_{16 \mathrm{~F}}=D_{1} G_{11}\left(R_{1}\right), \\
& L_{17 \mathrm{~F}}=0,
\end{aligned}
$$




$$
\begin{aligned}
& L_{26 \mathrm{~F}}=D_{2} G_{22}\left(R_{2}\right), \\
& L_{27 \mathrm{~F}}=-D_{2} G_{11}\left(R_{2}\right) .
\end{aligned}
$$

The radial displacements $W_{1 n}$ and $W_{2 n}$ are respectively given by Eqs. (39) and (40) in which $|L|=$ $\left|\boldsymbol{L}_{1}\right| \cdot\left|\boldsymbol{L}_{2}\right|$ is used instead of Eq. (41):

$$
\begin{aligned}
W_{1 n}\left(k_{z}, \omega\right)= & F_{1}^{\prime}\left|\begin{array}{ll}
L_{11} & L_{12} \\
L_{12} & L_{14}
\end{array}\right| /\left|L_{1}\right|, \\
W_{2 n}\left(k_{z}, \omega\right)= & -F_{1}^{\prime} L_{27 \mathrm{~F}}\left|\begin{array}{ll}
L_{11} & L_{12} \\
L_{12} & L_{14}
\end{array}\right| \\
& \cdot\left|\begin{array}{ll}
L_{21} & L_{22} \\
L_{22} & L_{24}
\end{array}\right| /\left|L_{1}\right| \cdot\left|L_{2}\right| .
\end{aligned}
$$

The form of Eq. (48) indicates that the inner shell shows the forced response independent of the existence of the entrained fluid and outer shell. In other word, Eq. (48) corresponds to the forced response of an immersed signle shell (i.e., the inner shell alone of a double shell) driven by a point force.

\subsection{General Characteristics of the Solution}

From Eqs. (39) and (40) [also from Eqs. (48) and (49)] we get the following relation common to both strong and weak couplings:

$$
W_{2 n}\left(k_{z}, \omega\right)=-\left[L_{27 \mathrm{~F}} W_{1 n}\left(k_{z}, \omega\right)\right] \cdot \frac{\left|\begin{array}{ll}
L_{21} & L_{22} \\
L_{22} & L_{24}
\end{array}\right|}{\left|L_{2}\right|} .
$$

Comparing Eq. (50) with Eq. (48), we know that the outer shell is driven by the apparent force

$$
F_{2}{ }^{\prime}=-L_{27 \mathrm{~F}} W_{1 n}\left(k_{z}, \omega\right) \text {. }
$$

Therefore, as general characteristics of the doubleshell vibration, we may state the followings:

1) The vibrational characteristics of the inner shell $W_{1 n}\left(k_{k}, \omega\right)$ should appear on the outer shell of the double shell through the fluidstructure coupling element $L_{27}$.

2) The vibrational characteristics of the outer shell itself should appear through the matrix $L_{2}$.

Next let us consider the phase of vibration. The determinant $\left(L_{21} L_{24}-L_{22}{ }^{2}\right)$ in Eq. (50) takes a real value. Another determinant $\left|L_{2}\right|$ becomes real if $c \leq c_{0}$ but complex if $c>c_{0}$ because of the propagator $G_{22}\left(R_{2}\right)$ of Eq. (25). The coupling element $L_{27 \mathrm{~F}}$ of Eq. (35) for strong coupling and of Eq. (47) for weak coupling takes a positive real value if $c \leq c_{0}$ is satisfied. ${ }^{13)}$ Hence, we may add the following statements on the phase of vibration:

3) In-phase coupled vibration occurs if the subsonic condition $\left(c \leq c_{0}\right)$ is satisfied and $\left(L_{21} L_{24}-L_{22}{ }^{2}\right) /\left|L_{2}\right|$ takes a negative value.

4) Out-of-phase coupled vibration occurs if the subsonic condition is satisfied and $\left(L_{21} L_{24}-\right.$ $\left.L_{22}{ }^{2}\right) /\left|L_{2}\right|$ takes a positive value.

5) Neither in-phase nor out-of-phase vibration occurs if the supersonic condition $\left(c>c_{0}\right)$ is satisfied.

\section{NUMERICAL CALCULATION OF DISPERSION CURVE}

Before calculating the $\mathrm{K}$-space radial displacement $W_{1 n}\left(k_{z}, \omega\right)$ [defined as Eq. (39) or (48)] or $W_{2 n}\left(k_{z}, \omega\right)$ [defined as Eq. (40) or (49)] numerically, structure-borne energy dissipation should be introduced into the above theory by treating Young's modulus as a complex quantity, namely

$$
E \rightarrow E(1-i \eta),
$$

where $\eta$ denotes the hysteresis loss factor. When using this complex Young's modulus, we make the following replacements in the equations described in the previous sections:

$$
c_{p}{ }^{2} \rightarrow c_{p}{ }^{2}(1-i \eta) \text { and } \Omega_{i}{ }^{2} \rightarrow \frac{\Omega_{i}{ }^{2}}{1-i \eta} .
$$

Introducing the structural loss, we can certainly avoid the infinity because the denominators of Eqs. (39), (40), (48), and (49) will not become null.

Now let us compare the vibration due to the strong coupling as shown in Fig. 3(a) with that due to the weak coupling as shown in Fig. 3(b). We will concentrate on the outer shell because the vibrational characteristics of the outer shell are much more important than those of the inner shell from the viewpoint of underwater radiation, and because the vibrational characteristics of the inner shell should appear on the outer shell as shown by Eq. (50). The logarithmic level $20 \log \left|W_{2 n}\left(k_{z}, \omega\right)\right|$ is then drawn in Fig. 4(a) for the strong coupling of Eq. (40) and in Fig. 4(b) for the weak coupling of Eq. (49), respectively. The circumferential wavenumber $n$ is fixed to 3,4 , and 5 for the left, middle, and right frames, respectively. The abscissa is the axial wavenumber $m$ which is treated as a continuous quantity according to Eq. (1). The ordinate is the frequency $f$ of vibration in $\mathrm{Hz}$. The curve shown in Fig. 4 is referred to as the dispersion curve since the 


\section{S. YOSHIKAWA: FLUID-STRUCTURE COUPLED VIBRATION OF DOUBLE SHELL}
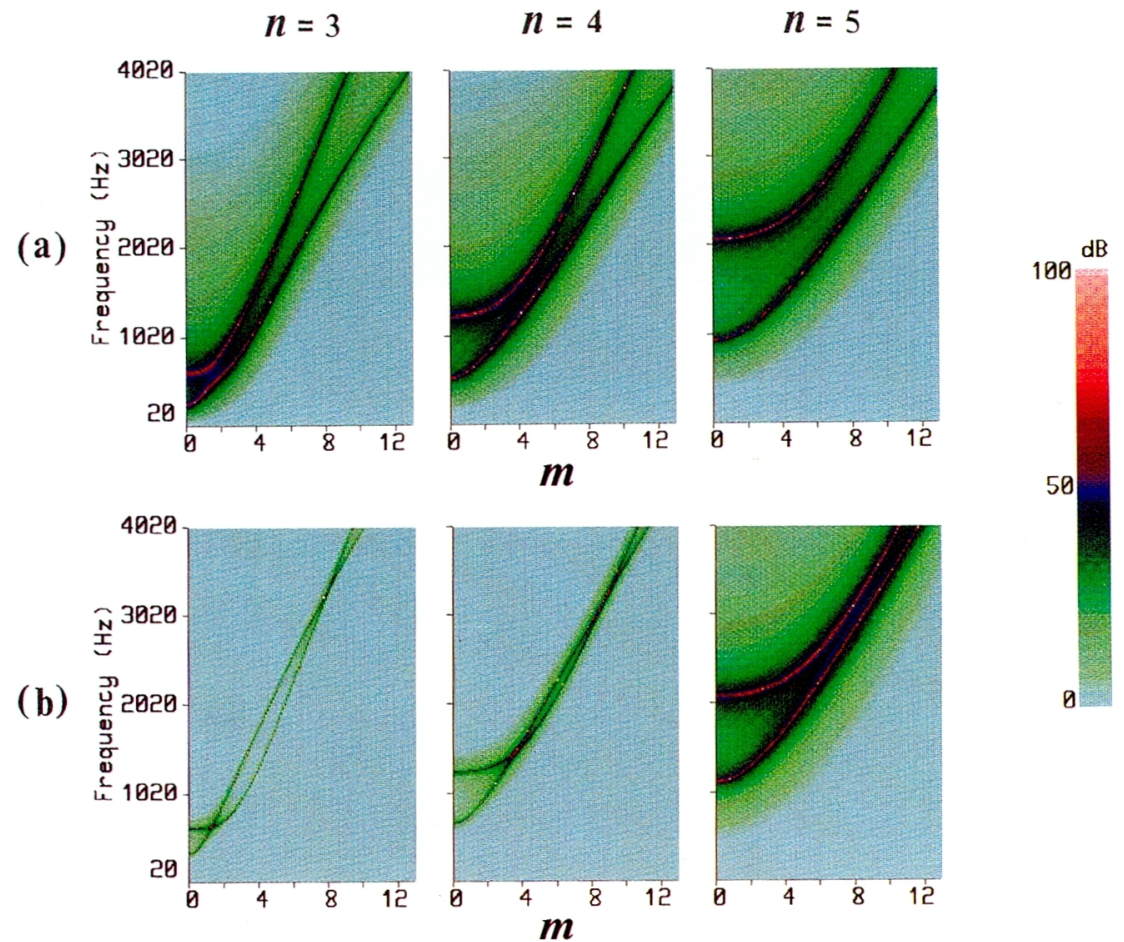

Fig. 4 Theoretical dispersion curve of the absolute radial displacement of the outer shell as a function of the axial mode number $m$ and frequency $f$. (a) strong coupling, (b) weak coupling.

value of $\omega / k_{z}$, which is given by a point $(m, f)$ on a curve, defines the local phase velocity $c$ of the helical wave in Eq. (13). For numerical calculation, parameter values listed in Table 1 were employed, and an appropriate value was input into the driving force $F_{1}$ in Eq. (36). The range of calculation was $20 \leq$ $f \leq 4,000 \mathrm{~Hz}$ (in $20-\mathrm{Hz}$ steps) and $0.00025 \leq m \leq 12.9$ (in 0.10 steps). The digital data obtained by this calculation is converted to the logarithmic one in which the largest value corresponds to $100 \mathrm{~dB}$. Such resulting data is displayed using color intensity to represent the logarithmic level: 32 shades of green for 0 to $50 \mathrm{~dB}$ and another 32 shades of red for 50 to $100 \mathrm{~dB}$. As the displacement amplitude approaches maximum, the color approaches white from red.

From Fig. 4 we may state the followings:

1) Both strong and weak couplings give two dispersion curves.

2) These two curves are well separated from each other in Fig. 4(a), while the lower curve ap- proaches to the upper one and intersects with it for $n=3$ and 4 in Fig. 4(b).

3) The upper curve which gives higher frequencies for the same $m$ [or gives higher cutoff frequency at $m=0$ for $n=3$ and 4 in Fig. 4(b)] looks almost identical with each other in Figs. 4(a) and 4(b).

4) The above 3) means that the upper dispersion curve suggests the forced response of the inner shell driven by a point force.

5) The lower dispersion curve then indicates the induced response of the outer shell and reflects the difference in the coupling by the entrained fluid.

6) Strong coupling moves the lower dispersion curve downwards more than weak coupling does.

Numerical calculation on the phase of vibration adds the following statements [see Ref. 4) for detailed numerical results]:

7) In-phase vibration happens on the upper dis- 


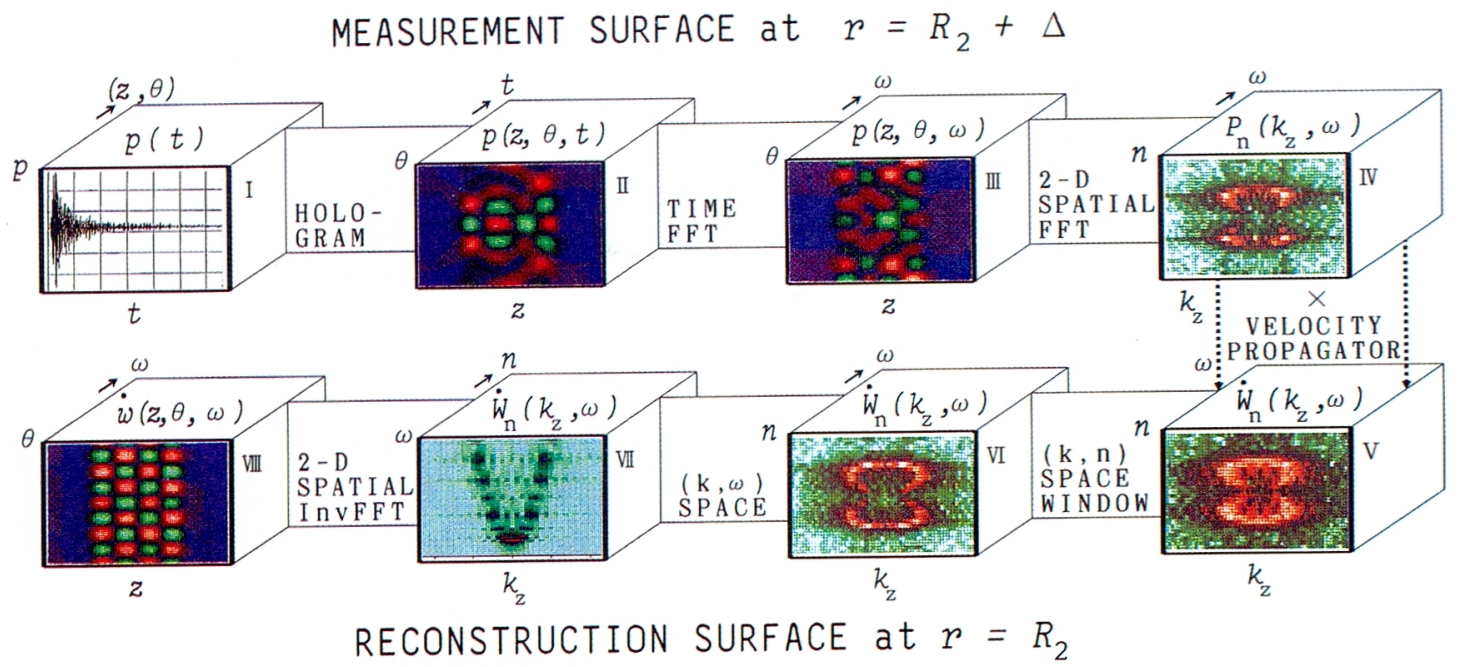

Fig. 5 Schematic of GENAH signal processing. An ensemble of acoustic pressure $p(t)$ at the grid points $(z, \theta)$ on the measurement surface (cf. frame I) reveals characteristic mode patterns $\dot{w}(z, \theta)$ of the vibrating surface for a given frequency range (cf. frame VIII). See text items 1) to 8) which correspond to frames I to VIII, respectively.
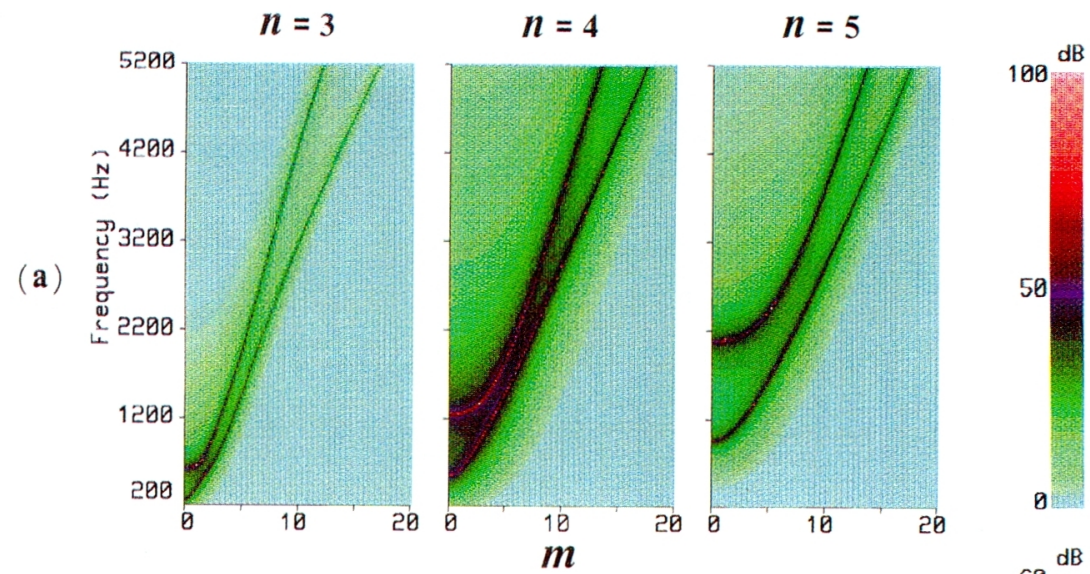

(b)
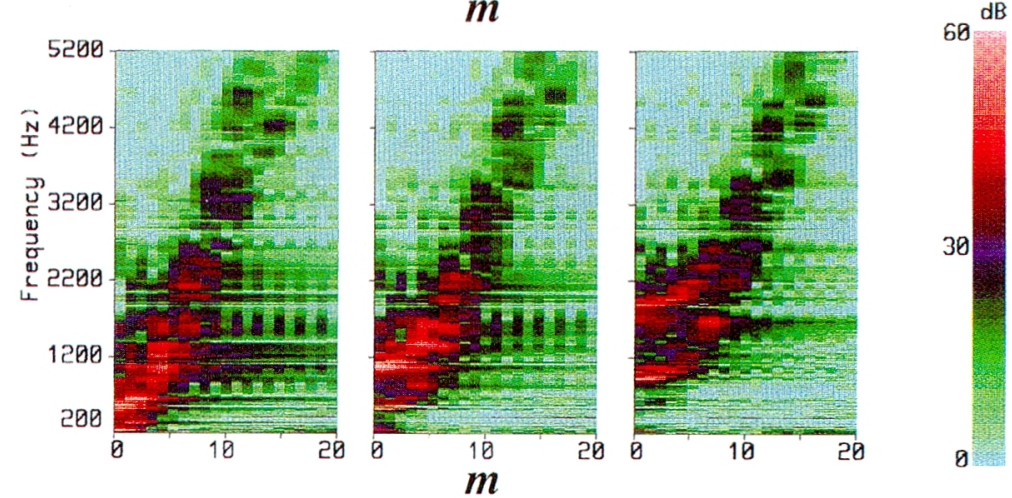

Fig. 6 Comparison of double-shell's dispersion curves. (a) strong-coupling theory, (b) GENAH experiment. 


\section{S. YOSHIKAWA: FLUID-STRUCTURE COUPLED VIBRATION OF DOUBLE SHELL}

persion curve.

8) Out-of-phase vibration happens on the lower dispersion curve.

These results indicate that items 3) and 4) stated in Section 6.3 are realized in the numerical calculation on our experimental model. Exactly speaking, if the structural loss is introduced, these items are not correct because two determinants and $L_{27 \mathrm{~F}}$ in Eq. (50) take complex values. However, as long as the loss factor is as small as 0.001 , it brings only negligible effect to the phase relation.

\section{BROADBAND GENAH EXPERIMENT AND DISCUSSION}

8.1 Outline of Generalized Near-field Acoustical Holography

The experiment was carried out in the U.S. Naval Research Laboratory's AARF (advanced acoustic research facility) pool. An automated hydrophone positioner has been developed for the experimental implementation of GENAH at this facility. The procedure of the broadband GENAH experiment ${ }^{8,9}$ is outlined below and shown in each frame (from I to VIII) of Fig. 5, where the case of single shell is illustrated for simplicity:

1) One hydrophone receives the radiated acoustic pressure due to the pulsed excitation with a chirp signal (a 10-ms pulse covering the frequency range of $100 \mathrm{~Hz}$ to $6 \mathrm{kHz}$ drove the shaker at a repitition rate of $1.0 \mathrm{~Hz}$ in our experiment). Analog pressure signal is sampled in very short (a few $10 \mu$ s) intervals. Sampling inception time is precisely controlled by a computer, which works as the reference source for holographic interference. 32 responses of the excitation are added to enhance the SN ratio. The same things are repeated on the preset measurement surface concentric to the vibrating cylindrical shell. This measurement surface was at a distance of $\Delta=20 \mathrm{~mm}$ from the shell surface $\left(r=R_{2}\right)$, twice as long as the shell, and divided into $64 \times 64$ grid points in our experiment.

2) Sequential data ensemble of acoustic pressure $p(t)$ at the grid points $(z, \theta)$ is converted to an ensemble of acoustic pressure distribution $p(z, \theta)$ over the measurement surface at each sampling time $t$. Such resulting data set is called acoustic pressure hologram.

3) Temporal Fourier transform is applied to this hologram.

4) Moreover, the two-dimensional, spatial Fourier transform is applied and we obtain another hologram with respect to wavenumber-frequency space. It is called $K$-space representation of the hologram.

5) This pressure hologram is back propagated from the measurement surface onto the vibrating surface, and transformed to K-space vibrational velocity hologram by multifying velocity propagator.

6) Appropriate window in wavenumber space is applied to this velocity hologram.

7) K-space dispersion curve is given by plotting $\dot{W}_{n}\left(k_{z}, \omega\right)$ as a function of $k_{z}$ and $\omega$ instead of $k_{z}$ and $n$.

8) Characteristic mode patterns $\dot{w}(z, \theta)$ of the vibrating surface for a given frequency range are reconstructed by applying the two-dimensional, spatial inverse Fourier transform. The surface velocity exhibits $(4,4)$ mode of $1,411 \mathrm{~Hz}$ in Fig. 5, where red and green colors correspond to 0 to 10 and 0 to -10 respectively, and white color to 10 or -10 in arbitrary unit.

\subsection{Comparison between Theory and Experiment}

Vibrational modes of the radial surface velocity obtained by the above GENAH experiment are shown in Fig. 5 of Ref. 4), Fig. 1 of Ref. 7), and Fig. 3 of Ref. 14). These figures indicate that the simply-supported boundary condition of Eq. (1) may be realized because the surface velocity approaches null near the shell ends. For clear comparison of theory with experiment, it is, however, much more persuasive to discuss on the basis of the dispersion curve rather than the vibrational mode. See Figs. 6 and 7 of Ref. 4) or Fig. 2 of Ref. 7) for detailed information of the experimental dispersion curves corresponding to the theoretical ones shown in Fig. 4(a) of this paper.

Figure 6 is prepared to examine which model of strong and weak couplings shows better agreement with the experimental results. Figure 6(a) shows $20 \log \left|W_{2 n}\left(k_{z}, \omega\right)\right|$ in which Eq. (40) of strong coupling was recalculated in the range of $200 \leq f \leq 5,200$ $\mathrm{Hz}$ (in $20-\mathrm{Hz}$ steps) and $0.00025 \leq m \leq 19.9$ (in 0.157 steps). Figure 6(b) shows $20 \log \left|\dot{W}_{2 n}\left(k_{z}, \omega\right)\right|$ of the experimentally obtained radial velocity of the outer shell of the double shell. Time-series data of 
the measured acoustic pressure consists of 1,024 points resulting in 512 frequency bins which start from $198 \mathrm{~Hz}$ in 11.4-Hz steps. On the other hand, 64 points were measured along the axial length which is twice as long as the shell, and then the scanning step of $25 \mathrm{~mm}$ determines the maximum value of $m$ as 32. The resolution of Fig. 6(b) is, however, relatively low because the plotting was done in one steps with respect to $m$. Also, the maximum value of color intensity corresponds to $60 \mathrm{~dB}$ because dynamic levels of experimental data were not so high. Moreover, in Fig. 6(b) you may notice many streaks which extend from the high-level, red points to either side of them laterally. This effect is recognized as an oscillation of a sinc function $\sin (x) / x$ centered at a point on a dispersion curve. This sinc function is due to the finite length of the shell, and derived by changing the infinite integral on $z$ in Eq. (4) into the finite integral bounded from $-l / 2$ to $l / 2$. See Ref. 15) for more detailed discussion on the effects of shell length and signal processing.

Comparing Fig. 6(a) with Fig. 6(b), we notice that the shape of theoretical dispersion curves based on the strong-coupling model is very similar to that of experimental ones. This general agreement between the theory and experiment proves that the strong coupling controls the coupled vibration of our experimental double shell.

\section{CONCLUSIONS}

Two kinds of theory on the vibration of a pointdriven "double shell" were proposed from the viewpoint of fluid-structure coupling. One of them is based on the strong coupling which forms a standing wavefield in the entrained fluid; The other is based on the weak coupling which forms a free wavefield in it. In each theory, $6 \times 6$ matrix equation with fluid-structure coupling elements was finally derived from Flügge's infinite-shell theory, Helmholtz' fluidfield equation, and boundary conditions given at the fluid-shell interfaces. The solution of this matrix equation for the coupled vibration, that is, the radial surface displacements of the inner and outer shells of a double shell were represented in wavenumberfrequency $(k-\omega)$ space.

Numerical calculation on the experimental model of a doutle shell revealed the following vibrational behaviors of the double shell:

1) Both strong and weak couplings in the double shell bring two separate dispersion curves.

2) One dispersion curve defines higher-frequency, in-phase vibration.

3) The other curve defines lower-frequency, outof-phase vibration.

4) The higher-frequency dispersion curve, which indicates the forced response of the inner shell, is almost the same whether the fluidstructure coupling is strong or weak.

5) The lower-frequency dispersion curve, which indicates the induced response of the outer shell, depends on the coupling strength: Strong coupling moves this curve downwards (to lower-frequency direction) more than weak coupling does.

The existence of two separate dispersion curves was confirmed by the GENAH experiment on the outer shell of our double-shell model. Experimental dispersion curves were very similar to theoretical ones based on the strong coupling. This agreement demonstrates the validity of strong-coupling theory on the double-shell vibration. This means that the reflection of acoustic wave takes place at the interface between the shell of stainless steel and the entrained water in our experimental model. We may hence comment that the outer shell of double-shell structure is not acoustically transparent but can shield sound radiation from the inner shell to some degree.

\section{ACKNOWLEDGEMENTS}

A part of this work was carried out in the U.S. Naval Research Laboratory between July 1990 and October 1991 under the US Navy Scientist Exchange Program. The author appreciates Mr. Bob Reynolds of the Navy International Program Office and Dr. Burton G. Hurdle (Code 5103) of NRL for their long-term support and help to realize the program. The author is also grateful to Dr. Earl G. Williams and Mr. Karl B. Washburn (Code 5137) of NRL for their sincere encouragement and proper advices for the research, to Dr. David L. Bradley (Code 5100) and Dr. Joseph A. Bucaro (Code 5130) for their support to conduct the research smoothly, and to Dr. Brain H. Houston and Mr. Roger Volk (Code 5136) who arranged and carried out the experiment. The author expresses special thanks to Mr. Kiyoshi Koyano of Izumi Engineering Laboratory Co., Ltd. and Mr. Mitsuto Ohshita of Sekishu Engineering Co., Ltd. for their support in building 


\section{S. YOSHIKAWA: FLUID-STRUCTURE COUPLED VIBRATION OF DOUBLE SHELL}

up the experimental model.

\section{REFERENCES}

1) M. C. Junger and D. Feit, Sound, Structures, and Their Interaction, 2nd Ed. (MIT Press, Cambridge, Mass., 1986).

2) F. Fahy, Sound and Structural Vibration (Academic Press, London, 1985).

3) S. Yoshikawa, T. Abe, H. Fujita, and K. Koyano, "Study on vibration and sound radiation of concentric double cylinder," Proc. Spring Meet. Acoust. Soc. Jpn. 3-8-7, 775-776 (1987) (in Japanese).

4) S. Yoshikawa, E. G. Williams, and K. B. Washburn, "Vibration of two concentric submerged cylindrical shells coupled by the entrained fluid," NRL Rep. (1991) (unpublished).

5) S. Yoshikawa, E. G. Williams, and K. B. Washburn, "Theory and experiment of two concentric submerged cylindrical shells coupled by the entrained fluid," J. Acoust. Soc. Am. 89, 6SA8, 1955 (1991).

6) S. Yoshikawa, "Coupled vibration of two concentric submerged cylindrical shells as a model of submersible's hull structure: Theory," Proc. Spring Meet. Acoust. Soc. Jpn. 2-8-9, 1035-1036 (1992) (in Japanese).

7) S. Yoshikawa, E. G. Williams, and K. B. Washburn, "Coupled vibration of two concentric submerged cylindrical shells as a model of submersible's hull structure: Experiment," Proc. Spring Meet. Acoust. Soc. Jpn. 2-8-10, 1037-1038 (1992) (in Japanese).

8) E. G. Williams, H. D. Dardy, and K. B. Washburn, "Generalized nearfield acoustical holography for cylindrical geometry: Theory and experiment," J. Acoust. Soc. Am. 81, 389-407 (1987).

9) E. G. Williams, B. H. Houston, and J. A. Bucaro, "Broadband nearfield acoustical holography for vibrating cylinders," J. Acoust. Soc. Am. 86, 674679 (1989).

10) Ref. 1) pp. 151-152.

11) A. W. Leissa, "Vibration of shells," NASA Rep. SP-288, Chap. 2 (1973).

12) Ref. 2) pp. 166-172, 186-191, 259-268.

13) According to the Handbook of Mathematical Functions with Formulas, Graphs, and Mathematical Tables, M. Abramowitz and I. A. Stegun, Eds.
(National Bureau of Standards, Washington, D.C., 1970), the numerator $I_{n}(b) K_{n}{ }^{\prime}(b)-K_{n}(b) I_{n}{ }^{\prime}(b)$ of Eq. (35), where $b=k_{r}{ }^{\prime} R_{2}$, equals $-1 / b$ (see formulas 9.6.15 and 9.6.26). On the other hand, the denominator $I_{n}{ }^{\prime}(a) K_{n}{ }^{\prime}(b)-K_{n}{ }^{\prime}(a) I_{n}{ }^{\prime}(b)<0$, where $a=$ $k_{r}{ }^{\prime} R_{1}$, because $I_{n}{ }^{\prime}(b)>I_{n}{ }^{\prime}(a)>0$ and $K_{n}{ }^{\prime}(a)<K_{n}{ }^{\prime}(b)$ $<0$ (see Fig. 9.7, formulas 9.6.7, 9.6.9, 9.7.3 and 9.7.4.)

14) S. Yoshikawa, "Joint research on the "coupled vibration of two concentric submerged cylindrical shells" conducted at the U.S. Naval Research Laboratory and brief introduction of the laboratory," J. Mar. Acoust. Soc. Jpn. 19, 123-131 (1992) (in Japanese).

15) E. G. Williams, B. H. Houston, and J. A. Bucaro, "Experimental investigation of the wave propagation on a point-driven, submerged capped cylinder using K-space analysis," J. Acoust. Soc. Am. 87, 513-522 (1990).

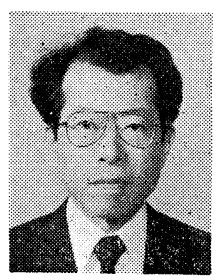

Shigeru Yoshikawa was born in Ebina, Kanagawa, on December 3, 1949. He received the B. Sci. in physics in 1974 from Nagoya University and the M. Eng. in electronics in 1978 from Tamagawa University. He also studied musical acoustics at Kyushu Institute of Design from 1974 to 1976, and underwater acoustics at Tokyo Institute of Technology in 1979. He was conferred the Dr. Eng. in 1985 from Tokyo Institute of Technology. He joined the 5th Research Center, Technical R \& D Institute, Defense Agency in 1980, where he is currently directing the research as a Section Head. He was appointed as a Visiting Research Professor at the U.S. Naval Academy in 1988 and an Exchange Scientist at the U.S. Naval Research Laboratory in 1990. His research interests focus on the physics of organ pipes and other musical wind instruments, underwater sound projectors, and underwater structural acoustics and vibration. $\mathrm{He}$ is a member of the Acoustical Society of Japan, the Acoustical Society of America, the Marine Acoustics Society of Japan, and the Japan Society of Fluid Mechanics. He was awarded the 21st Sato Memorial Paper Prize in 1981 from ASJ. 